\title{
Saturated Orientational Polarization of Polar Molecules in Giant Electrorheological Fluids
}

\author{
P. Tan, W. J. Tian, X. F. Wu, J. Y. Huang, L. W. Zhou,* and J. P. Huang* \\ Department of Physics and Surface Physics Laboratory (National Key Laboratory), Fudan University, \\ Shanghai 200433, China
}

Received: December 27, 2008; Revised Manuscript Received: May 9, 2009

\begin{abstract}
Many researches on polar-molecular electrorheological (PMER) fluids with giant electrorheological effects were reported in recent years. The particles of PMER fluids (PMER particles) are known to have a dielectric core with high dielectric constant and a shell of polar molecules. Our calculation of local electric fields using the finite element approach shows that the local electric field can cause an orientational polarization of the polar molecules. The saturation of the orientational polarization occurs on the outer shells of two nearby PMER particles. Then, it causes the strong outer shell-outer shell interaction between the two particles, and this kind of interaction is just responsible for the giant electrorheological effect. It is further realized that the PMER effect is mainly due to the interaction of the tail-head connected polar molecules within the two outer shells between the two PMER particles. Our theoretical results of static yield stresses are shown to be in excellent agreement with the reported experimental data by several groups. For general PMER fluids, the calculated static yield stress is nearly proportional to $R^{x-1}$. When $h / R$, the ratio between the thickness of shells and radius of PMER particles, changes from 0.05 to 0.5 , the index $x$ changes accordingly from 0.64 to 0.51. It is also found that particles with thinner thickness $h$ and smaller radius $R$ have larger electrorheological effects until the static yield stress shows a peak when $R$ reaches about $10 \mathrm{~nm}$.
\end{abstract}

\section{Introduction}

Electrorheological (ER) fluids are suspensions composed of polarizable colloidal particles dispersed in an insulating carrier fluid. ${ }^{1-20}$ They can be transformed into solidlike state almost instantaneously $(1-10 \mathrm{~ms})$ when an electric field is applied, and the transition is reversible. This phenomenon is expected to be widely used in industries, for example, in active control of conventional and intelligent devices. Recently, the structure and mechanisms of ER fluids have been carefully reviewed in an elegant article by Wen, Huang, and Sheng. ${ }^{21}$ The physical mechanism of dielectric ER fluids has been well established, of which the upper bounds on the static yield stress varies as $1.38\left(R_{1} / \delta_{1}\right)^{1 / 2}$ in units of $\varepsilon_{\mathrm{f}} E_{0}{ }^{2} / 8 \pi .^{22-24}$ Here $R_{1}$ denotes the radius of suspended dielectric particles, $\delta_{1}$ the surface-to-surface separation between two neighboring particles, $\varepsilon_{f}$ the dielectric constant of a host liquid, and $E_{0}$ an external electric field. The upper bounds of the static yield stress is proportional to $E_{0}^{2}$ and has a value of $8 \mathrm{kPa}$ when $R_{1}=20 \mu \mathrm{m}, \delta_{1}=0.1 \mathrm{~nm}, E_{0}$ $=1 \mathrm{kV} / \mathrm{mm}, \varepsilon_{\mathrm{f}}=2.5 \varepsilon_{0}$, and the ratio $\varepsilon_{\mathrm{p}} / \varepsilon_{\mathrm{f}}$ approaches infinity. ${ }^{24}$ Here $\varepsilon_{\mathrm{p}}$ denotes the dielectric constant of the particles.

In recent years several polar-molecular electrorheological (PMER) fluids have been fabricated, ${ }^{20,25,26}$ whose static yield stresses exceed the predicted upper bounds of dielectric ER fluids significantly. The static yield stress of these reported PMER fluids shows a linear relation with the external applied electric field. The size of these reported ER particles is tens or hundreds of nanometers. Many aspects of these ER fluids violate the induced polarization model of dielectric ER fluids. These PMER particles were fabricated to have polar molecules coated, modified, or absorbed on a dielectric core (Figure 1). (Hereafter we use "PMER particles" to denote dielectric cores coated with a shell of polar molecules suspended in PMER fluids.) The

* To whom correspondence should be addressed. E-mail: 1wzhou@ fudan.edu.cn; jphuang@fudan.edu.cn.

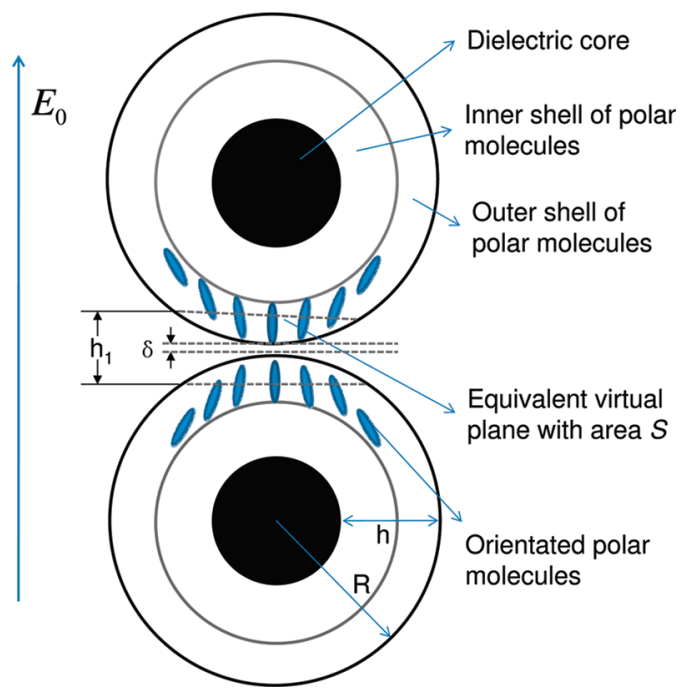

Figure 1. Schematic graph showing two touching spherical PMER particles with a dielectric core and a shell of polar molecules. The shell is composed of an inner part and an outer one. The outer shell has a thickness of a single polar molecule. In this outer shell, the polar molecules are orientated by the external electric field $E_{0}$. In the inner shell, the polar molecules are seen to be randomly oriented since they are more difficult to orient than those in the outer shell (its reason can be found in the text content). The equivalent virtual plane with area $S$ for simplifying outer shell-outer shell interaction is also indicated, as used in eq 7. Some symbols like $R, h, \delta$, and $h_{1}$ are also indicated. The graph is not shown to scale.

core-shell structure was also observed experimentally and supported by the dielectric measurement. ${ }^{25}$ Many researches of the surface properties of these particles show there exist a lot of multivalent ions on the surface of the dielectric cores, and this gave further evidence to the core-shell structure because there exists ion-dipole interaction between polar molecules and 


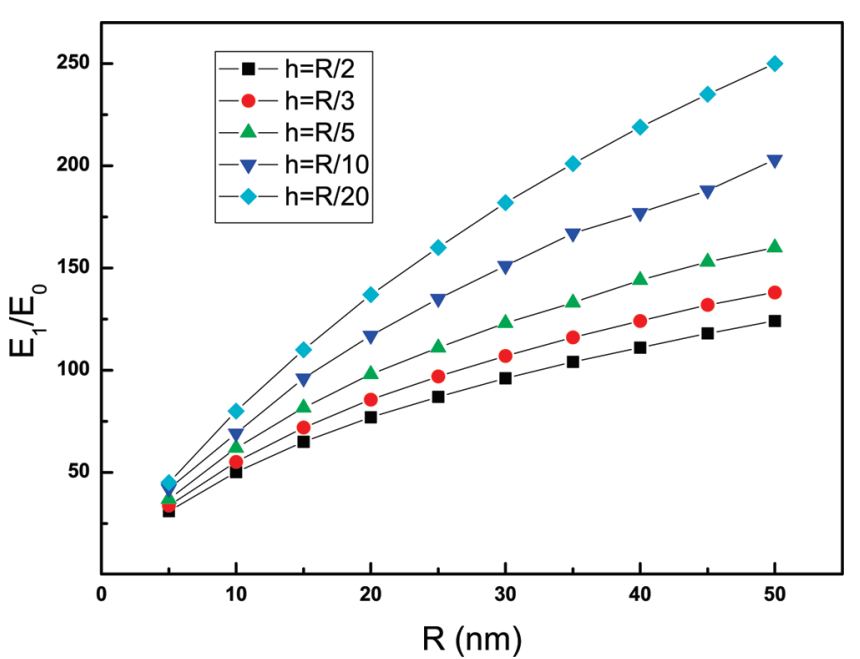

Figure 2. $E_{1} / E_{0}$ as a function of $R$ for different shell thickness $h$ at $\delta$ $=0.2 \mathrm{~nm}$, calculated by using the FEA. Other parameters in use have been indicated in the text content.

ions on the surface of dielectric cores. An induced-polarization saturation model was first proposed in refs 23 and 25 to explain the aspects of PMER fluids. The elastic contact of PMER particles was proposed in order to obtain accordance with the experiments in the literature. ${ }^{20,26}$ When the shell of polar molecules is thick, the shell elasticity plays a role in the interaction between PMER particles, and it may become negligible for thin shells of polar molecules.

Following the pioneering works by Wen, Sheng, and their coauthors, ${ }^{23,25}$ the purpose of this work is to determine theoretically the role of polar molecules in the interaction between PMER particles. We develop a model by proposing that the orientational polarizability of the polar molecules in a shell is saturated when a local electric field is above a certain value. The calculation of the local electric field distribution using the finite element approach (FEA) proves that the local electric field in a small area on the outer shells of two nearby PMER particles is large enough to cause the orientational polarization of polar molecules to be saturated (Figure 2). Then the interaction between two PMER particles is considered as that between two polarized outer shells of polar molecules under appropriate conditions. The calculated results of static yield stresses show good agreement with the reported experimental data by several groups. Thus this supports the model in which the orientational polarization of polar molecules is responsible for the large ER effect. It is also found that PMER particles with thinner shell and smaller radius have larger ER effects until the static yield stress shows a peak when the radius of PMER particle reaches about $10 \mathrm{~nm}$. This indicates, when the PMER-particle radius is smaller than this range, the local electric field would not be large enough to cause the saturation of orientational polarization of the polar molecules.

\section{Orientational Polarization of Polar Molecules in PMER Fluids}

A. Polarizability of Polar Molecules and Local Electric Field Enhancement between Two nearby PMER Particles in an Infinite Particle Chain. Let us start by considering a PMER composite particle which has a core/shell structure. The dielectric core has a large dielectric constant while the shell is composed of polar molecules. The particle is polarized under an applied electric field. In principle, for such cases, the electric field distribution in and around the composite particle can be analytically solved by solving the Maxwell equations together with appropriate boundary conditions which are related to the dielectric constants of the core/shell and the host medium. The induced dipole moment $\mu_{\text {induced }}$ of a polar molecule acquired in a field $E$ is determined by the sum of its electronic induced dipole moment and orientational dipole moment, namely, $\vec{\mu}_{\text {induced }}$ $=\vec{\mu}_{\text {electronic }}+\vec{\mu}_{\text {orient }}$. Here the $\mu_{\text {electronic }}$ arises from the electronic displacements in molecules. Based on a simple one-electron atom model of polar molecules, ${ }^{27} \mu_{\text {electronic }}$ is given by

$$
\mu_{\text {electronic }}=\frac{1}{2} \pi \varepsilon_{0} d^{3} E
$$

where $d$ is the diameter of a polar molecule and $\varepsilon_{0}$ the dielectric constant of free space. Throughout this work, we set $d=0.45$ $\mathrm{nm}$ if there are no special instructions. The orientational dipole moment $\mu_{\text {orient }}$ originates from the effect of an external electric field on the Boltzmann-averaged orientations of dipoles. Its contribution averaged over all angles $\Theta$ leads to

$$
\begin{aligned}
\mu_{\text {orient }} & =\langle\mu \cos \Theta \exp (\mu E \cos \Theta / \mathrm{kT})\rangle \\
& =\mu\left(\frac{e^{\alpha}+e^{-\alpha}}{e^{\alpha}-e^{-\alpha}}-\frac{1}{\alpha}\right) \\
& \equiv \mu L_{\alpha}(E)
\end{aligned}
$$

where $\alpha=\mu E / k T, \mu$ is the permanent dipole moment of a polar molecule, $k$ the Boltzmann constant, and $T$ the temperature.

The polarizability of the shell of polar molecules is related with the local field strength. We divided the shell into two parts, the inner shell (which is assumed to be a solid coating) and the outer shell (within which the polar molecules are assumed to be able to rotate freely in the absence of an external electric field). The equivalent dielectric constant of the inner shell can be readily estimated as

$$
\varepsilon_{\text {innershell }}=1+\frac{n \mu_{\text {induced }}}{\varepsilon_{0} E}
$$

where $n$ is the number density of polar molecules. In this equation, we may assume $\mu_{\text {orient }} \approx \mu^{2} E / 3 k T$ for $\mu E \ll k T$ owing to the screening effect arising from the outer shell. According to the dielectric property measurement of PMER particles, they have an effective (real) dielectric constant of 60 for urea molecules in direct current (DC) cases. ${ }^{25}$ It is worth mentioning that only DC cases are considered and calculated throughout this work. Regarding the outer shell, we assume that it contains only one single layer of polar molecules for simplicity. Under this assumption, both the elasticity and induced polarizability of the outer shell can be small enough to be neglected, and the orientational polarizability of the outer shell is described by eq 2. We first discuss the contribution of the dielectric core and the inner shell to the local electric field in order to get the polarizability of the outer shell. The local electric field computation was discussed in a series of papers, ${ }^{28-31}$ in which the multipole-expansion theory and the FEA were adopted to treat similar systems which contain large mismatches in the dielectric constants (and conductivities) at the surfaces of the spheres and/ or shells. In contrast to some accurate theories like the Rayleigh method, ${ }^{32,33}$ the FEA is accurate and convenient in describing the electrostatics of dielectric materials and quasi-statics of dielectric and conducting materials. Thus, in this work we shall 
resort to the FEA. In details, we use instead the FEA, which has been implemented in the commercial software COMSOL Multiphysics 3.5 , to numerically simulate the system. The unit cell which contains two adjacent particles in an infinite particle chain of the PMER fluids is treated as the lattice and the boundary condition is that there is a voltage difference between the two parallel plates of the unit cell which is perpendicular to the direction of the applied electric field. The voltage difference is given by the field strength times $4 R+2 \delta$, as indicated in Figure 1. The crucial governing equation is $-\nabla \varepsilon_{0} \varepsilon_{\mathrm{r}} \nabla \Phi=0$ in each domain. Here $\varepsilon_{\mathrm{r}}$ denotes a relative dielectric constant of the material, and $\Phi$ is the electric potential. The electric field distribution (inside and around the composite particle) in the cell can be calculated by setting the appropriate material parameters and boundary conditions as required by the software. Figure 2 displays the electrostatic properties of PMER particles, with parameters setting as follows: the relative dielectric constant of the dielectric core $\varepsilon_{\text {core }}=1000$, the relative dielectric constant of the (inner and outer) shell of polar molecules $\varepsilon_{\text {shell }}=60$, and the relative dielectric constant of the dispersed liquid or silicone oil $\varepsilon_{\mathrm{f}}=2.5$. All the parameters used in the calculations accord with real systems. The modulus of the local electric field between two touching PMER particles along the direction of the external electric filed $E_{0}$ is calculated, and the results of the local electric field enhancement $E_{1} / E_{0}$ with different $R$ (radius of PMER particles) and $h$ (thickness of shells) are plotted in Figure 2. Here $E_{1}$ represents the maximum value of the local electric field between the two PMER particles. It is worth mentioning that the $E_{1}$ only includes the induced polarization of the dielectric core and the inner shell of the polar molecules (Figure 1), and that it does not contain the influence of orientational polarization of the outer shell of polar molecules. Throughout this work, the gap $\delta$ between two nearby PMER particles is set to be $0.2 \mathrm{~nm}$.

According to the FEA calculation results, an estimated relation between the local field $E_{1}$ and the external electric field $E_{0}$ for different sizes of PMER particles can be represented by $E_{1}=\beta E_{0}$, where $\beta$ denotes a local-field enhancement factor determined by the dielectric property of PMER fluids. As can be seen in Figure 2, smaller $h$ leads to larger $\beta$. Meanwhile, smaller $R$ causes smaller $\beta$.

For PMER particles with radius $R$ of tens of nanometers, the field enhancement in the gap between them is about $\beta=100$ according to the FEA. Normally when the external electric field is $1 \mathrm{kV} / \mathrm{mm}, E_{1}$ can exceed $100 \mathrm{kV} / \mathrm{mm}$. The orientational polarization $P$ of the outer shell in such a local field can be described by the Langevin function $L_{\alpha}(E)$, and $P=n \mu L_{\alpha}(E)$, where $E$ should include $E_{1}$ and the contribution from the nearby outer shell.

Next, we use $E_{\text {local }}$ to denote the maximum local electric field at the outer shell of a PMER particle, at least approximately. By considering all the contributions (specifically, the polarization of polar molecules can affect in turn the local electric field) which may occur at the poles of the PMER particle, we may express $E_{\text {local }}$ in a self-consistent equation like

$$
E_{\text {local }}=E_{1}+L_{\alpha}\left(E_{\text {local }}\right) \frac{n \mu}{\varepsilon_{\mathrm{f}}}
$$

By numerically solving eq 4 , the relation between $E_{\text {local }}$ and $E_{1}$ is shown in Figure 3. Apparently, $L_{\alpha}\left(E_{\text {local }}\right)$ quickly becomes saturated with the increase of $E_{1}$, which means the saturation of the orientational polarization, and, after the saturation of $L_{\alpha}\left(E_{\text {local }}\right), E_{\text {local }}$ increases linearly with $E_{1}$.

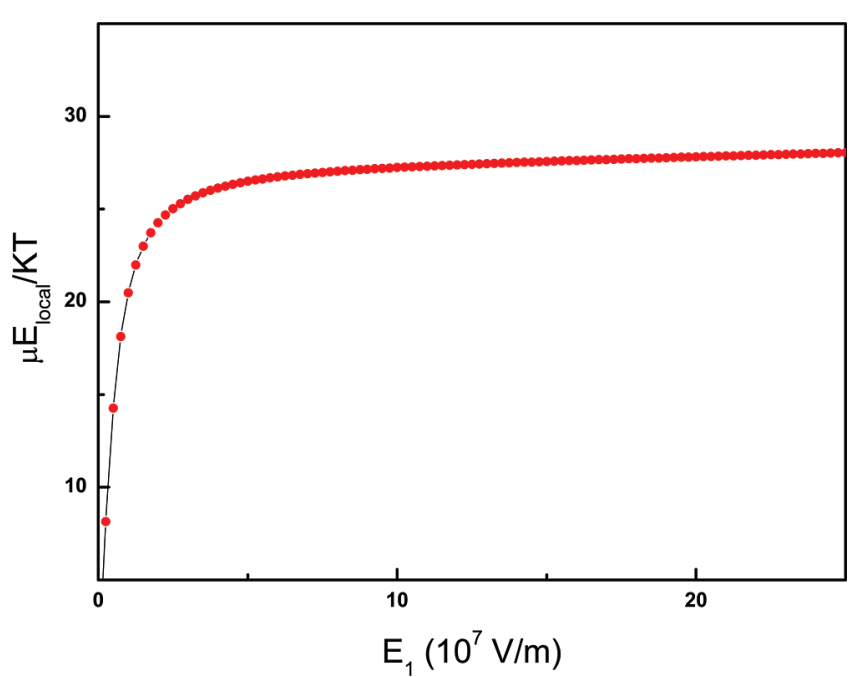

Figure 3. Reduced electrostatic energy at a pole (within the outer shell of urea polar molecules) of a PMER particle as a function of $E_{1}$ according to eq 4. Parameters: $\mu=4.56 \mathrm{D}$ and $T=300 \mathrm{~K}$.

B. Interaction between Two PMER Particles. The electrostatic interaction between two nearby PMER particles generally consists of three parts: (i) the polar molecule-polar molecule interaction, (ii) the dielectric core-dielectric core interaction, and (iii) the interaction between one dielectric core and the polar molecules on the other dielectric core. Here part ii was fully discussed in a series of papers, and it can not be the origin of the giant electrorheological effect. ${ }^{22,24}$ And part iii cannot be the origin either, partly due to the screening of polar molecules. Now let us focus on (i) the polar molecule-polar molecule interaction. This interaction is just between two polarized outer shells, namely, outer shell-outer shell interaction. The polarization $P$ of the outer shell is determined by the local electric field. By the definition of an electric field distribution function $f(\theta), E_{\text {local }}(\theta)=E_{\text {local }} f(\theta)$ (Here $\theta$ is an angle with respect to the external electric field), the electrostatic energy of the polarized outer shell (with a thickness of a single polar molecule, see Figure 1) is given by

$$
U=2 \pi R^{2} d \int_{0}^{\pi / 2} \mathbf{P} \cdot \mathbf{E}_{\text {local }}(\theta) \sin \theta \cos \theta \mathrm{d} \theta
$$

Here $\mathbf{E}_{\text {local }}(\theta)$ means the local electric field on the outer shell at the angle $\theta$, and it is to be extracted from the calculated results of the electric field distribution by a fitting with a Lorentz function. Then, we simply use the Lorentz function to replace $f(\theta)$ in the following calculations [Figures 4-6]. According to eqs 4 and 5 , eq 5 can be written as

$$
U=2 \pi R^{2} \int_{0}^{\pi / 2} n \mu d L_{\alpha}\left(E_{\text {local }} f(\theta)\right) E_{\text {local }} f(\theta) \sin \theta \cos \theta \mathrm{d} \theta
$$

The number density $n$ of polar molecules is approximately $1 / d^{3}$.

For the sake of convenience, we may further set the interaction between two PMER particles to be equivalent to an interaction between two small virtual planes of polar molecules, each of which has an area $S$ (as indicated in Figure 1)

$$
S=2 \pi R^{2} \int_{0}^{\pi / 2} \frac{L_{\alpha}\left(E_{\text {local }} f(\theta)\right) f(\theta) \sin \theta \cos \theta}{L_{\alpha}\left(E_{\text {local }}\right)} \mathrm{d} \theta
$$




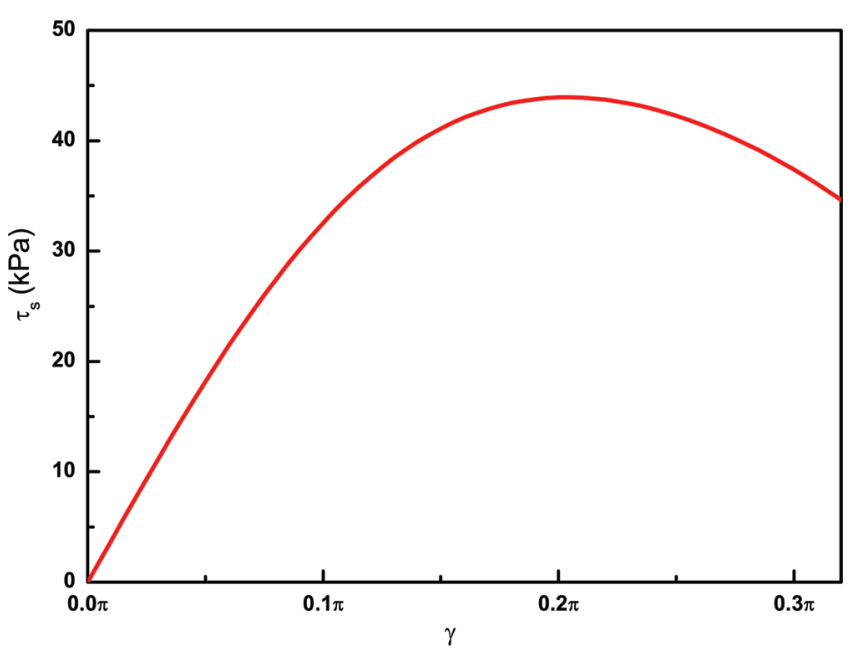

Figure 4. Relation between shear stress $\tau_{\mathrm{s}}$ and strain $\gamma$ of PMER fluids according to eq 9. Parameters: $R=25 \mathrm{~nm}, E_{0}=2 \mathrm{kV} / \mathrm{mm}, \mu=4.56$ $\mathrm{D}$, and $\phi=30 \%$.

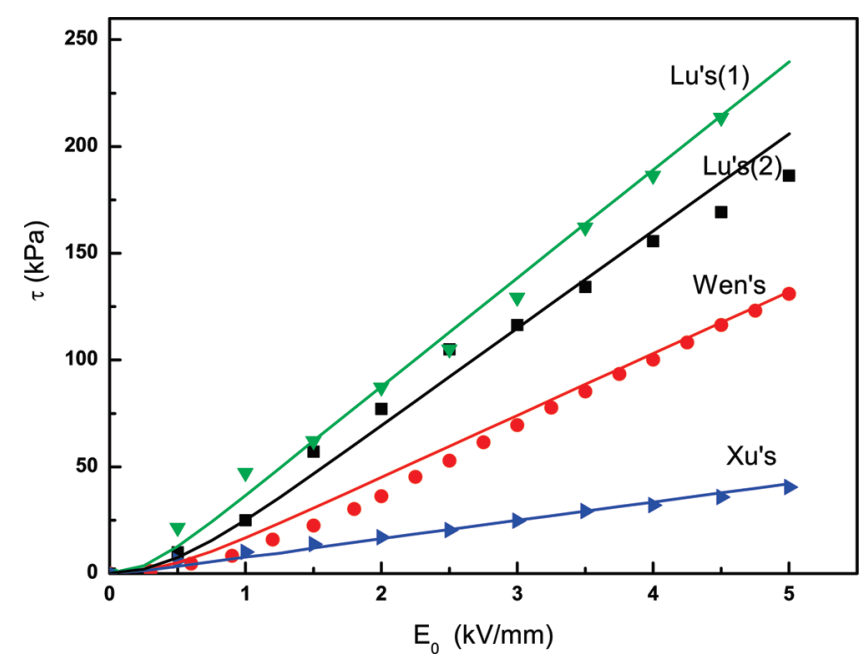

Figure 5. Static yield stress $\tau$ of PMER fluids as a function of $E_{0}$ : A comparison between experimental data (symbols) and corresponding theoretical results (lines). The parameters used in the theoretical calculations based on eq 9 have been listed in Table 1 .

and the distance between the two virtual planes is

$$
h_{1}=\frac{2 S}{\pi R}+\delta
$$

On the virtual plane, the polarization and local electric field are identical everywhere [eq 7], thus simplifying the research. The local field at the virtue plane has been set to be $E_{\text {local }}$, as already implied by $L_{\alpha}\left(E_{\text {local }}\right)$ in eq 7 . Equation 7 has been derived by considering the fact that the electro-static energy of the virtual plane should be equal to that of the corresponding PMER particle. It is worth noting that eq 7 was obtained by considering $\theta \in[0, \pi / 2]$. Actually, the influence from $\theta \in[\pi / 2, \pi]$ can be small enough to be neglected due to the screening effect.

Now the shear stress $\tau_{\mathrm{s}}$ of a PMER fluid is given by

$$
\tau_{\mathrm{s}}=\frac{F_{\gamma}}{S_{\mathrm{p}}}=\frac{\partial U}{S_{\mathrm{p}} h_{1} \partial \gamma}
$$

where $S_{\mathrm{p}}=\pi(2 R)^{2} / 6 \phi$ is the average section area occupied by a chain of PMER particles, $\phi$ is the volume fraction of PMER

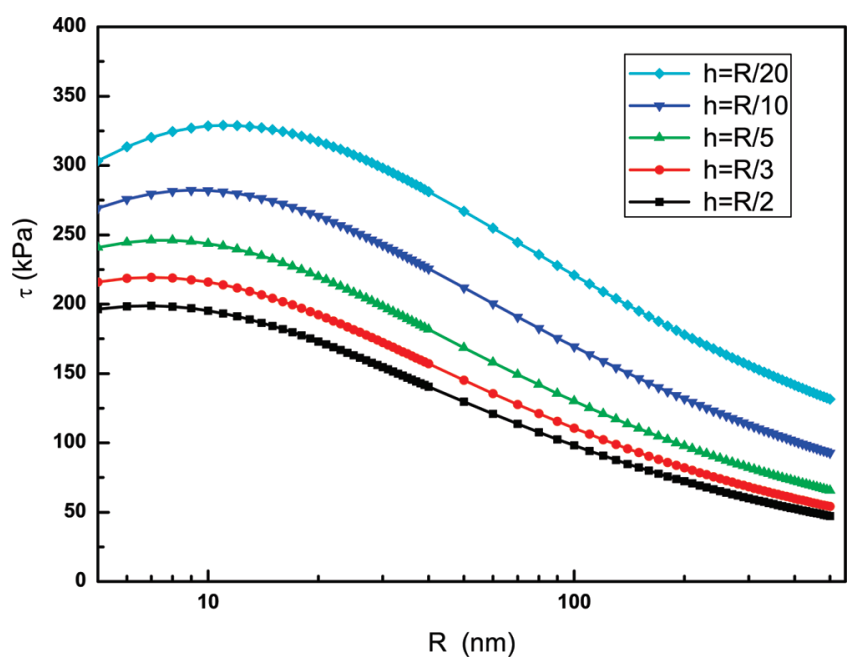

Figure 6. Static yield stress $\tau$ of PMER fluids versus $R$ for various $h$, according to eq 9 . The local field enhancement factors $\beta$ used in the calculation are extracted from Figure 2. Other parameters in use are the same as those used in Figure 2 as well.

TABLE 1: Parameters ${ }^{20,25,26}$ Adopted in the Calculation of Static Yield Stress, as Shown in Figure 5

\begin{tabular}{llcccccc}
\hline & \multicolumn{1}{c}{ shell } & $d(\mathrm{~nm})$ & $\mu(\mathrm{D})$ & $R(\mathrm{~nm})$ & $\beta$ & $\delta(\mathrm{nm})$ & $\phi$ \\
\hline Wen's $^{25}$ & $\left(\mathrm{NH}_{2}\right)_{2} \mathrm{CO}$ & 0.45 & 4.56 & 30 & 80 & 0.2 & 0.3 \\
${\text { Lu's }(1)^{26}}^{\left(\mathrm{NH}_{2}\right)_{2} \mathrm{CO}}$ & 0.45 & 4.56 & 32.5 & 130 & 0.2 & 0.35 \\
${\text { Lu's }(2)^{26}}^{\mathrm{C}_{2} \mathrm{H}_{5} \mathrm{NO}}$ & 0.45 & 3.76 & 32.5 & 129 & 0.2 & 0.35 \\
Xu's $^{20}$ & $\mathrm{C}_{4} \mathrm{H}_{6} \mathrm{O}_{2}$ & 0.36 & 4.524 & 450 & 170 & 0.2 & 0.35
\end{tabular}

particles, and $\gamma$ the tilted angle of a chain. To obtain $\tau_{\mathrm{s}}$, we use the FEA to determine the relation between $E_{\text {local }}$ [eq 4] and $\gamma$, by assuming that the second term in the right-hand side of eq 4 is independent of $\gamma$ due to the saturation of the orientational polarization. It is realized that the major contribution of eq 9 is from the integration of $\theta \in[0,0.17]$. Namely, the PMER effect is mainly due to the interaction of the tail-head connected polar molecules within the two outer shells between the two PMER particles. Now we can get the stress-strain relation of PMER materials. A typical stress -strain relation at $E_{0}=2 \mathrm{kV} / \mathrm{mm}$ is plotted in Figure 4. At a certain tilted angle $\gamma$, the shear stress reaches its maximum $\tau$ (namely, static yield stress). The large value of $\tau$ originates mainly from the large saturated orientational polarizability of the outer shell at the poles of the PMER particles and $\tau \propto \partial E_{\text {local }} / \partial \gamma \propto \partial E_{1} / \partial \gamma \propto E_{0}$ after $L_{\alpha}\left(E_{\text {local }}\right)$ is saturated, as can be inferred from eqs $4-6$. This can explain the experimentally revealed linear relation between $\tau$ and $E_{0}$ for various kinds of PMER fluids (such experimental data have been displayed in Figure 5). For this purpose, Figure 5 shows the comparison between our theoretical calculations and experimental data. Good agreement has been shown. The parameters used in the calculations are listed in Table 1. All of the parameters, except for the fixed $\delta(=0.2 \mathrm{~nm})$ and the corresponding value of $\beta$, are directly extracted from refs 20 , 25 , and 26 accordingly. For the fitting, the $\beta$ was obtained according to the FEA, see Figure 2.

C. Properties of PMER Fluids Caused by Orientational Polarization of Polar Molecules. As mentioned above, the large value of $\tau$ originates mainly from the saturated orientational polarization of the outer shell of polar molecules. In other words, once the head-to-tail structure is formed near the poles of two nearby PMER particles, it is hard to break down, thus leading to large $\tau$.

We may also investigate the size effect of PMER fluids. It can be seen from the derived equation, the shear stress is 
TABLE 2: $E_{1} / E_{0}$ Calculated by the FEA for Both DC and AC Cases with Various Low Frequencies ${ }^{a}$

\begin{tabular}{lc}
\hline \multicolumn{1}{c}{ cases } & $E_{1} / E_{0}$ \\
\hline $\mathrm{DC}$ & 71.234 \\
$\mathrm{AC}(5 \mathrm{~Hz})$ & 71.292 \\
$\mathrm{AC}(10 \mathrm{~Hz})$ & 71.246 \\
$\mathrm{AC}(20 \mathrm{~Hz})$ & 71.235 \\
$\mathrm{AC}(30 \mathrm{~Hz})$ & 71.233 \\
$\mathrm{AC}(40 \mathrm{~Hz})$ & 71.232 \\
$\mathrm{AC}(50 \mathrm{~Hz})$ & 71.231
\end{tabular}

${ }^{a}$ For the DC case, the conductivity of the dielectric core $\sigma_{\text {core }}$, the conductivity of the (inner and outer) shell $\sigma_{\text {shell }}$, and the conductivity of the dispersed liquid or silicone oil $\sigma_{\mathrm{f}}$ are set to be zero, respectively. For all the AC cases, $\sigma_{\text {core }}=10^{-11} \mathrm{~S} / \mathrm{m}, \sigma_{\text {shell }}=10^{-9}$ $\mathrm{S} / \mathrm{m}$, and $\sigma_{\mathrm{f}}=10^{-13} \mathrm{~S} / \mathrm{m}$. Other parameters: $R / \delta=50, \varepsilon_{\text {core }}=1000$, $\varepsilon_{\text {shell }}=60$, and $\varepsilon_{\mathrm{f}}=2.5$. All the parameters accord with real systems.

proportional to $\beta / R$, when $\delta$ is much smaller than $S / R$. So PMER particles with small size $R$ will have better ER effects when the polarization is saturated. The PMER fluids have a size effect different from that of dielectric ER fluids because $\beta$ may vary with the size of PMER particles and the thickness of the shell. In our simulation in Figure 2, we find $\beta \propto R^{x}$, where $x=0.64$, $0.59,0.54,0.52$, and 0.51 for $h=R / 20, R / 10, R / 5, R / 3$, and $R / 2$, respectively. Then the static yield stress $\tau \propto R^{x-1}$ according to eq 9. Figure 6 displays the size effect of PMER fluids with different shell thickness $h$. One can see that PMER particles with thinner shell and smaller radius yield a larger static yield stress $\tau$ until the $\tau$ reaches a peak. That is to say, the $\tau$ does not increase infinitely with decreasing $R$. We found that such a peak comes to appear at radius $R=10 \mathrm{~nm}$ or so. As a model example, within the sense of an infinitesimally thin inner shell of polar molecules, we take urea molecules to be the polar molecules inside the outer shell, and the static yield stress is calculated to be $628.5 \mathrm{kPa}$ at $5 \mathrm{kV} / \mathrm{mm}$ when $R=10 \mathrm{~nm}$ and $\delta=0.2 \mathrm{~nm}$. Now we are in a position to summarize the physical reason for the appearance of peaks on stress-radius curves as shown in Figure 6. In general, PMER particles with smaller particle radius $R$ have better ER effects because the saturation of the orientational polarization of polar molecules in outer shells can be more easily achieved. However, with further decrease of $R$, the local electric field will be not strong enough to cause the saturation of the orientational polarization of the polar molecules. This will then lead to a reduction of the orientational polarization of the polar molecules. Thus, the static yield stress can be smaller as $R$ decreases. In this sense, a peak appears on such stressradius curves.

\section{Discussion and Conclusions}

Throughout this work, we have taken into account the mismatch of dielectric constants on boundaries accordingly by considering the DC case, for which the conductivities have actually been taken to be zero. To display the effects of the mismatch of conductivities, here we also investigate the alternating current (AC) cases with some low frequencies (which are available for conventional ER fluids), in comparison with the above-mentioned DC case. The comparison can be found in Table 2. It is evident that the mismatch of conductivities has almost no effect on the local electric field $E_{1}$ at low frequencies, and thus the desired yield stresses.

We have used an approximation method to calculate the shear stress. The main rationality of doing so lies in the following facts: The unit cell which contains two (adjacent) particles used in our calculations is located in an infinite particle chain of PMER fluids, and the boundary condition on each surface of the lattice is the same as that in real systems. It is worth mentioning that, to simulate an infinite particle chain of our interest, the detailed number of particles adopted in a unit cell has no evident effect on the accuracy of the calculated results for the parameter set in our use. Nevertheless, if the particles in the unit cell are close enough, both/either multipolar interaction between them ${ }^{34}$ and/or many-body effects ${ }^{35}$ could offer a correction. In addition, our method is by no means the only way for calculating the shear stress. For instance, one may also calculate the shear stress by considering some threedimensional periodic structures like the body-centered tetragonal lattice, see ref 24 .

To sum up, the saturated orientational polarization of a large number of polar molecules makes the pair interaction between PMER particles approximate to an outer shell-outer shell interaction. This is supported by the local electric field calculation using the FEA and the static yield stress which shows an excellent agreement between our theoretical results and the experimental data reported by several groups. Some major properties of PMER fluids has been achieved. For instance, PMER particles with thinner shell and smaller radius have larger ER effect. However the size of PMER particles should not be too small. Or else, the orientational polarization would not be able to reach saturation. Also, the shell of polar molecules can not be infinitesimally thin. Thus it may possibly lead to an upper limit of the static yield stress for PMER fluids.

Acknowledgment. This work is supported by the National Natural Science Foundation of China under Grant Nos. 10334020, 10574027, 10604014 and 10874025, the Shanghai Education Committee and the Shanghai Education Development Foundation ("Shu Guang" project under Grant No. 05SG01), and Chinese National Key Basic Research Special Fund under Grant No. 2006CB921706.

\section{References and Notes}

(1) Winslow, W. M. J. Appl. Phys. 1949, 20, 1137.

(2) Halsey, T. C. Science 1992, 258, 761.

(3) Gast, A. P.; Zukoski, C. F. Adv. Colloid Interface Sci. 1989, 30 , 153.

(4) Jordan, T. C.; Shaw, M. IEEE Trans. Electr. Insul. 1989, $24,849$.

(5) Zukoski, C. F. Annu. Rev. Mater. Sci. 1993, 23, 45.

(6) Weiss, K. D.; Carlson, J. D.; Coulter, J. P. J. Intell. Mater. Syst. Struct. 1993, 4, 13.

(7) Parthasarathya, M.; Klingenberg, D. J. Mater. Sci. Eng. R: Rep. 1996, 17, 57.

(8) Halsey, T. C.; Toor, W. Phys. Rev. Lett. 1990, 65, 2820

(9) Tao, R.; Sun, J. M. Phys. Rev. Lett. 1991, 67, 398.

(10) Clercx, H. J. H.; Bossis, G. Phys. Rev. E 1993, 48, 2721.

(11) Tao, R.; Jiang, Q. Phys. Rev. Lett. 1994, 73, 205.

(12) Wen, W. J.; Ma, H. R.; Tam, W. Y.; Sheng, P. Phys. Rev. E 1997, 55, R1294.

(13) Goodwin, J. W.; Markham, G. M.; Vincent, B. J. Phys. Chem. B 1997, 101, 1961

(14) Wan, J. T. K.; Yu, K. W.; Gu, G. Q. Phys. Rev. E 2000, 62, 6846.

(15) Wan, J. T. K.; Gu, G. Q.; Yu, K. W. Phys. Rev. E 2001, 63, 052501.

(16) Yin, J. B.; Zhao, X. P. J. Phys. Chem. B 2006, 110, 12916.

(17) Cao, J. G.; Huang, J. P.; Zhou, L. W. J. Phys. Chem. B 2006, 110, 11635 .

(18) Fang, Z. N.; Xue, H. T.; Bao, W.; Yang, Y.; Zhou, L. W.; Huang, J. P. Chem. Phys. Lett. 2007, 441, 314.

(19) Zhao, X. P.; Wang, B. X.; Li, J. J. Appl. Polym. Sci. 2008, 108, 2833

(20) Xu, L.; Tian, W. J.; Wu, X. F.; Cao, J. G.; Zhou, L. W.; Huang, J. P.; Gu, G. Q. J. Mater. Res. 2008, 23, 409.

(21) Wen, W. J.; Huang, X. X.; Sheng, P. Soft Matter 2008, 4, 200.

(22) Ma, H. R.; Wen, W. J.; Tam, W. Y.; Sheng, P. Adv. Phys. 2003, 52,343 .

(23) Huan, X. X.; Wen, W. J.; Yang, S. H.; Sheng, P. Solid State Commun. 2006, 139, 581 
(24) Ma, H. R.; Wen, W. J.; Tam, W. Y.; Sheng, P. Phys. Rev. Lett. 1996, 77,2499

(25) Wen, W. J.; Huang, X. X.; Yang, S. H.; Lu, K. Q.; Sheng, P. Nat. Mater. 2003, 2, 727.

(26) Lu, K. Q.; Shen, R.; Wang, X. Z.; Sun, G.; Wen, W. J.; Liu, J. X. Chin. Phys. 2006, 15, 2476.

(27) Israelachvili, J. N. Intermolecular and Surface Forces, 2nd ed.; Academic press: London, 2000; $\mathrm{p} 69$.

(28) Davis, L. C. J. Appl. Phys. 1992, 72, 1334.

(29) Davis, L. C. J. Appl. Phys. 1997, 81, 1985.
(30) Tao, R.; Jiang, Q.; Sim, H. K. Phys. Rev. E 1995, 52, 2727.

(31) Gonon, P.; Foulc, J.-N.; Atten, P.; Boissy, C. J. Appl. Phys. 1999, 86,7160 .

(32) Rayleigh, L. Philos. Mag. 1892, 34, 481.

(33) Gu, G. Q.; Yu, K. W.; Hui, P. M. Phys. Rev. B 1998, 58, 3057.

(34) Ju, Y.; Huang, J. P. J. Phys. Chem. B 2008, 112, 7865.

(35) Shen, M.; Cao, J. G.; Xue, H. T.; Huang, J. P.; Zhou, L. W. Chem. Phys. Lett. 2006, 423, 165.

JP8115116 